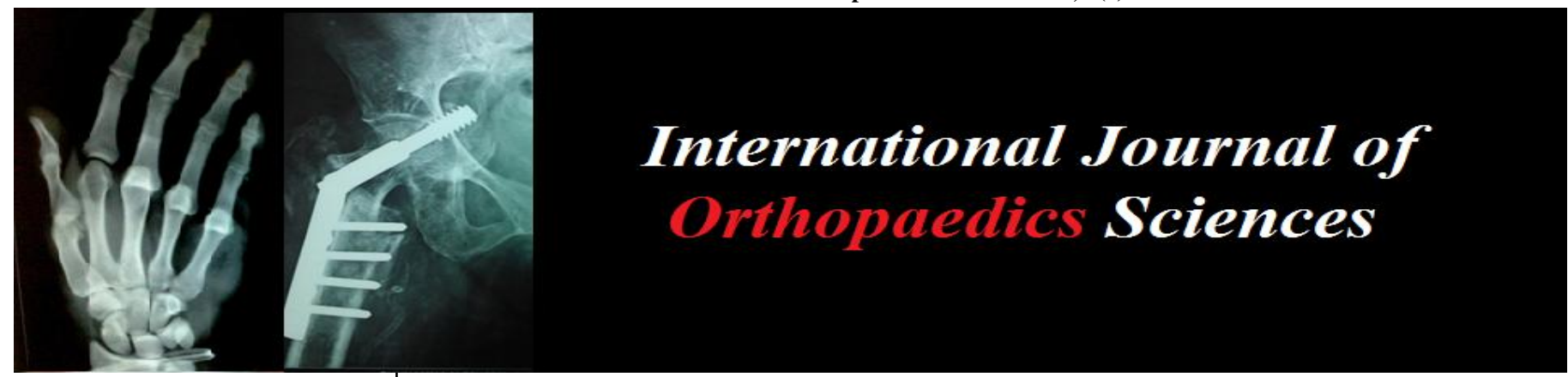

ISSN: $2395-1958$

IJOS 2018; 4(4): 40-45

(C) 2018 IJOS

www.orthopaper.com

Received: 23-08-2018

Accepted: 26-09-2018

Mr. Raviprasad Kattimani Specialty Doctor in Trauma and Orthopaedics, Warwick hospital Warwick, United Kingdom

Dr. Sowmya Sadanandappa Specialty Doctor in Obstetrics and Gynaecology, Warwick hospital, Warwick, United Kingdom

Mr. Chetan M Dojode Depuy Fellow in Shoulder and Knee surgery, East Surrey hospital, Redhill, United Kingdom

Dr. Umesh R Dixit

Associate Professor in

community Medicine, SDM

college of Medical Sciences

Dharwad, India

Mr. Gandavaram Srikant Reddy Specialty Doctor in Trauma and Orthopaedics, Lancaster Royal Infirmary, Lancaster, United Kingdom

Mr. Dhritiman Bhattacharjee Specialty Doctor in Trauma and Orthopaedics, Glan Clwyd hospital, United Kingdom

Mr. Amit Sinha

Consultant in Trauma and Orthopaedics, Glan Clwyd hospital, United Kingdom

Correspondence

Mr. Raviprasad Kattimani Specialty Doctor in Trauma and Orthopaedics, Warwick hospital Warwick, United Kingdom

\section{Medial parapatellar versus subvastus approach in total knee arthroplasty}

\author{
Mr. Raviprasad Kattimani, Dr. Sowmya Sadanandappa, Mr. Chetan M \\ Dojode, Dr. Umesh $\mathrm{R}$ Dixit, Mr. Gandavaram Srikant Reddy, Mr. \\ Dhritiman Bhattacharjee, Mr. Amit Sinha
}

DOI: https://doi.org/10.22271/ortho.2018.v4.i4a.10

\section{Abstract}

Background: Component alignment is one of the most important factors for long term survival after total knee arthroplasty. The medial parapatellar and subvastus are two commonly used surgical approaches in total knee arthroplasty.

Methods: The primary aim of this study was to compare component alignment between medial parapatellar and subvastus approach in total knee arthroplasty. The secondary aims were to analyse blood loss, duration of surgery, pain score in post-operative period, length of stay and post-operative complications. A retrospective study was undertaken comparing age and gender matched 60 total knee arthroplasty operated by either medial para patellar (30) or sub vastus approach (30) from January 2009 to December 2010 by two surgeons in a district general hospital, followed up to one year. Revision knee arthroplasty and total knee arthroplasty operated by other surgeons were excluded.

Student's t test and $\mathrm{Z}$ test were used for statistics.

Results: Subvastus approach had better component alignment in sagittal plane of tibia, coronal plane of femur and tibia $(\mathrm{p}<0.01)$. No difference in component alignment at sagittal plane of femur $(\mathrm{p}>0.05)$.

Pain was better controlled in subvastus approach on post-operative day three $(p<0.01)$. Operative time was more in subvastus group $(\mathrm{p}<0.05)$. There were no difference between two groups in duration of stay, blood loss and post-operative complications ( $\mathrm{p}>0.05)$.

Conclusion: Subvastus can be considered as an alternative surgical approach along with standard medial parapatellar approach for primary total knee arthroplasty.

Keywords: Medial parapatellar, subvastus, total, knee, arthroplasty

\section{Introduction}

Total knee arthroplasty is an effective treatment for advanced knee arthritis providing significant pain relief and improved joint function ${ }^{[1]}$

The success of total knee arthroplasty is dependent on many factors. Among all factors, component alignment is one of the most important factors for long term implant survival [2,3]. The Swedish knee arthroplasty registry noted that aseptic loosening and instability in components were the two most common indications for revision in total knee arthroplasty ${ }^{[4]}$.

The most common surgical approach for total knee arthroplasty is medial para patellar approach which is used as a standard approach in majority of knee joint replacement. ${ }^{[5,6]}$. It has the advantage of good joint exposure but also has drawbacks of impairing the extensor mechanism of knee joint and interfering with the vascular supply of patella. This results in complications, like patellar fracture, subluxation and avascular necrosis of patella. ${ }^{[5,6,7]}$

To overcome this disadvantage, Subvastus approach was re-introduced to the English speaking countries by Hoffman in $1991^{[8]}$. The advantages of this approach are that it's a quadriceps sparing procedure resulting in better postoperative knee range of movements and less impairment of vascular supply to patella. However the disadvantages were technical difficulty in exposure and eversion of patella. As it requires more technical skills, its popularity over medial para patellar approach was limited ${ }^{[9]}$. 


\section{Material and methods}

This study included sixty total knee arthroplasties which were performed at a district general hospital in North Wales for advanced osteoarthritis from 2009 to 2010. There were 30 knees in each group. They were selected randomly from theatre operating list operated by two different specific orthopaedic surgeons either by subvastus or medial para patellar approach. Both surgeons had more than ten years of experience in total knee arthroplasty.

Rheumatoid arthritis patients, Total knee arthroplasty operated by other surgeons, Unicondylar knee replacement, constrained prosthesis and conversion of high tibial osteotomy to total knee replacement were excluded from the study.

Patients were admitted to the elective orthopaedic ward after having total knee arthroplasty. Both groups of patients had pre-operative administration of intravenous antibiotic Ceftriaxone 1.5 grams at the time of induction of anaesthesia, followed by three doses post operatively. Intravenous Tranexamic acid 1 gram was given in both approaches at the time of induction.

All cases were operated in Laminar flow theatre. Bear hugger and mechanical leg pumps were used during intraoperative period for both group of patients. Tourniquet was used for all the patients inflated at time of incision and deflated after the application of compression dressing. Both the subvastus and medial parapatellar approach used advanced medial pivot knee system (Microport orthopaedic Inc, Arlington, Tennessee).

Both groups had similar pain management protocol. Mild pain was treated with paracetamol and non-steroidal antiinflammatory drugs, weak opioids like codeine were used for moderate pain and stronger opioids like morphine were administered for severe pain. Three further doses of intravenous antibiotics were given post operatively in both groups. Thrombo prophylaxis was carried out for 15 days postoperatively using subcutaneous low molecular weight heparin injections. All the patients received the same postoperative rehabilitation protocol. They were mobilised on post-operative day one with full weight bearing protocol by assistance from the physiotherapist using the walking frame.

\section{Radiological assessment}

Femur and tibia coronal alignment was evaluated by short antero posterior radiographs in weight bearing position with knee extended and patella pointing straight forward. In the lateral view knee was in extension with beam directed laterally and perpendicular to the knee joint. This method of imaging was followed for post-operative total knee arthroplasty radiographs.

\section{Coronal alignment of tibia and femur component}

The angle between anatomical axis of the femur and a line tangential to the distal condyles of the femoral component determines femoral varus or valgus alignment. Whereas, angle between the anatomical axis of the tibia and a line tangential to the plateau of the tibial component measures varus or valgus alignment of tibia. Tibial component should be placed in neutral alignment, which was 90 degrees ${ }^{[10]}$.

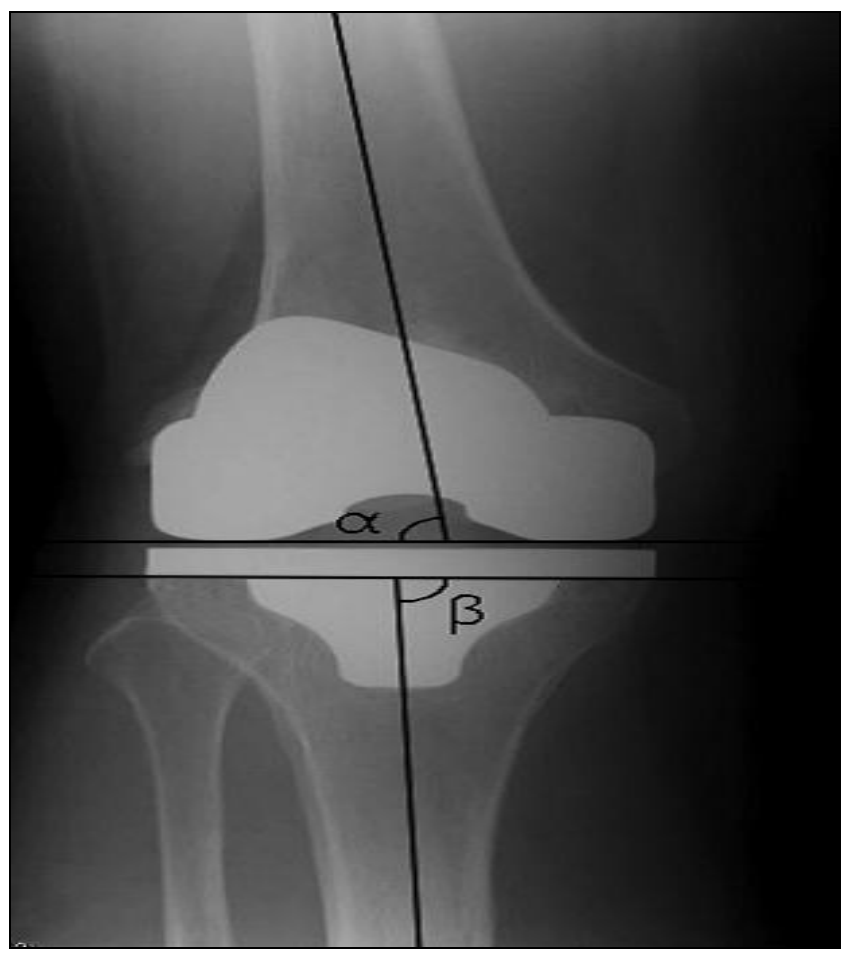

Fig 1: Measurement of coronal alignment of tibia and femur components

\subsection{Sagittal alignment of tibia and femur component}

The sagittal alignment of femur can be either in flexion or extension. Flexion of the femoral component was measured as the angle between the line across the bottom of the femoral implant and the femoral shaft axis. FF (flexion of femoral component) of 90 degrees corresponds to neutral placement, if it was more than 90 degrees corresponds to femoral component in extension, and if less than 90 corresponds to femoral component in flexion. The acceptable range of alignment was considered as 90 degrees +/- 3 degrees Tibial component alignment or tibial slope (TS) was measured as the angle between the line across the bottom of the tibial plate and the tibial shaft axis. TS of 90 degrees correspond to neutral placement, TS of more than 90 degrees corresponds to anterior tibial slope, whereas less than 90 corresponds to posterior tibial slope. (Fig 2) 


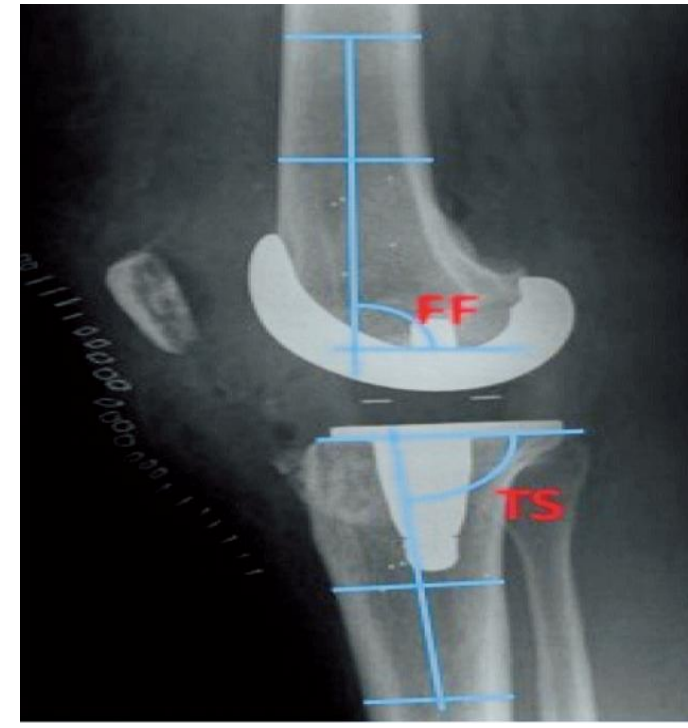

Fig 2: Measurement of sagittal alignment of tibia and femur components.

Blood loss was calculated from the pre-operative haemoglobin and post-operative check of haemoglobin twenty fours after procedure and also by looking into number of blood transfusions in each patient after surgery. According to local hospital guidelines, blood transfusion was done if haemoglobin level was less than $7 \mathrm{mg} / \mathrm{dl}$ (milligrams/decilitre) postoperatively or presence of signs and symptoms of cardiac insufficiency.
Pain scores were collected from patient daily observation chart at $8 \mathrm{AM}$; on post-operative day one and three using the visual analogue score (VAS). The scores ranged from 0 to 100. Score of 0 indicated least pain and 100 for the worst pain perceived. VAS was measured by asking the patient to mark a line on the VAS chart. Using this chart the score was determined by measuring the distance in millimetres on a ten centimetre line between no pain (0) and worst pain (100) as it is one of reliable assessment for accuracy of pain assessment [12].

Duration of surgery was calculated as time interval between inflation of tourniquet (at the time of surgical incision) to deflation of tourniquet. Duration of stay was time interval from date of surgery to date of discharge from hospital. Data regarding complications was collected by reviewing case notes, operative notes and clinic follow up notes.

\section{Statistics}

IBM SPSS (Statistical Package for the Social Sciences) software version 20 was used for interpretation of the results. Further analysis and application of statistical test was based on the type of data and the question to be answered. The value to cater type 1 error was set at 0.05 , so that any result with $\mathrm{p}$ value equal or less than 0.05 was considered as significant. In our study we used $\mathrm{Z}$ test and $\mathrm{t}$ test for the analysis of continuous variables.

\section{Results}

6.1 Basic demographics

Table 1: Basic demographics

\begin{tabular}{|c|c|c|c|c|c|}
\hline Surgical approach & Age & \multicolumn{2}{|c|}{ Sex } & \multicolumn{2}{c|}{ Limb side } \\
\hline & & female & male & Right & Left \\
\hline Subvastus & 67.9 & $63.3 \%$ & $37.6 \%$ & $56.6 \%$ & $43.3 \%$ \\
\hline Medial parapatellar & 65.4 & $66.7 \%$ & $33.3 \%$ & $53.3 \%$ & $47.3 \%$ \\
\hline
\end{tabular}

\subsection{Component alignment}

Subvastus approach had better component alignment in sagittal plane of tibia, coronal plane of femur and tibia.

Table 2: Component alignment

\begin{tabular}{|c|c|c|c|c|c|}
\hline & \multicolumn{2}{|c|}{ Subvastus(n=30) } & \multicolumn{2}{c|}{ Medial para patellar $(\mathbf{n = 3 0})$} & P value \\
\hline & Mean & SD+/- error & Mean & SD+/- error & \\
\hline Coronal femur & 91.83 & $4.77+/-0.8$ & 89.93 & $4.02+/-0.74$ & $>0.5$ \\
\cline { 2 - 6 } Coronal Tibia & 90.57 & $3.54+/-0.64$ & 88.48 & $2.53+/-0.47$ & $<0.01$ \\
\hline Sagittal Femur & 85.67 & $6.3+/-1.16$ & 81.93 & $2.53+/-0.47$ & $<0.01$ \\
\cline { 2 - 6 } Sagittal Tibia & 88.43 & $3.3+/-0.60$ & 85.93 & $3.116+/-0.57$ & $<0.01$ \\
\hline Tibio Femoral axis & 5.80 & $2.57+/-0.47$ & 4.17 & $1.80+/-0.32$ & 0.003 \\
\hline
\end{tabular}

\subsection{Coronal alignment}

The coronal alignment of tibia was significantly better in subvastus group when compared to medial parapatellar ( $\mathrm{p}$ <0.01). However, there was no statistical significant difference in coronal alignment of femur, between the two approaches $(p>0.05)$. In both groups the coronal alignment was in expected range of $90+/-3$ degrees.

Coronal tibial component alignment in subvastus group was mean 90.57 degrees (range 86 to 103 degrees) and in medial parapatellar group it was 88.48 degrees (range 82 to 94 degrees). The femoral component mean was 91.83 (80 to 105 degrees) in subvastus and 89.9 degrees in other group (81 to 96 degrees).

\subsection{Sagittal alignment}

Both femur and tibial alignment in sagittal plane were better in subvastus group $(\mathrm{p}<0.01)$. The component alignment of the femur in sagittal plane for 30 knees in subvastus group revealed the mean angle to be 85.67 degrees (range 76 to 102 degrees). The medial parapatellar group had a mean of 81.93 degrees (range 76-88). The ideal femoral component positioning in sagittal plane was considered to be 83 degrees of flexion to 90 degrees of neutral alignment ${ }^{[11]}$.

The sagittal alignment of tibia was posterior tibial slope of 88.43 degrees (range 79-93). Whereas, in medial para patellar it was 85.93 degrees (range $80-94$ ). It was better in subvastus group $(\mathrm{p}<0.01)$. The accepted range of tibial component placement in sagittal plane is between 83 to 90 degrees ${ }^{[11]}$.

\section{Clinical outcomes 7.1 Blood loss}

There was no statistical significant difference between the two groups in terms of blood loss and blood transfusions ( $p>0.05$ ). It was measured by drop in the haemoglobin calculated in 
$\mathrm{gm} / \mathrm{dl}$ (grams per decilitre) from preoperative period to twenty four hours post-operatively. Mean drop in the haemoglobin in subvastus group was $3.53 \mathrm{gm} / \mathrm{dl}$. Range was from $1.6 \mathrm{gm} / \mathrm{dl}$ to $5.7 \mathrm{gm} / \mathrm{dl}$ (SD: 1.08). Whereas, mean drop in haemoglobin in patients operated by standard approach was $3.15 \mathrm{gm} / \mathrm{dl}$ (SD: 1.009).

\subsection{Operative time}

There was statistical significant difference between two groups in the operative time $(\mathrm{p}<0.05)$. Subvastus had more operative time with mean duration of surgery of 91.60 minutes, (range 56 - 135) whereas; the mean operative time was 71.7 minutes (range 40 - 127) in medial para patellar approach.

\subsection{Pain score}

Pain was less in subvastus approach group. Post operatively pain was measured on day 1 and day 3 using visual analogue score. On day 1 in the medial parapatellar group 40 percent of patients had score of 0 . Whereas in subvastus approach 56.7 percent had score of 0 on day 1 but the mean score was 1.5 and 1.7 respectively. There was no statistical significant difference between two groups with respect to pain on day 1 . On day 3, in the subvastus group 33 percent of patients experienced pain score of 0 and only 13.3 percent had a score of 0 in patients operated by medial parapatellar approach. Mean pain score was 2.5 and 3.4 in subvastus and medial para patellar group respectively. Pain score on day 3 was significantly less in subvastus group when compared to medial para patellar group ( $\mathrm{p}$ value $<0.01$ ).

\subsection{Duration of stay in hospital}

Although Subvastus group (6.67 days) had shorter duration of stay in the hospital (range of 3- 11 days) than medial parapatellar group (8.03 days) (range of 4 to 14 days). There was no statistical significant difference between two groups in terms of duration of stay ( $p>0.05)$.

Table 3: Clinical outcomes

\begin{tabular}{|c|c|c|c|c|}
\hline & $\begin{array}{c}\text { Subvastus(n=30) Mean } \\
(\mathrm{SD}+/ \text { - error })\end{array}$ & $\begin{array}{c}\text { Medial parapatellar }(\mathbf{n}=30) \\
\text { Mean (SD +/- error) }\end{array}$ & $\begin{array}{c}\mathrm{Z} \\
\text { score }\end{array}$ & $\begin{array}{c}\mathbf{P} \\
\text { value }\end{array}$ \\
\hline Haemoglobin $(\mathrm{Hb})$ difference (pre op -post op) in $(\mathrm{gm} / \mathrm{dl})$ & $3.53(1.08+/-0.19)$ & $3.15(1.009+/-0.18$ & 1.42 & $>0.5$ \\
\hline Blood transfusion & 6 & 7 & 1.85 & $>0.5$ \\
\hline Operative time (Minutes ) & $91.60(22.17+/-4.0)$ & $71.17(19.66+/-3.5)$ & 3.78 & 0.001 \\
\hline Length of stay (Days) & $6.67(1.68+/-0.38)$ & $8.03(3.46+/-0.63)$ & 1.94 & 0.058 \\
\hline Pain score on Day 1 & $1.5(2.16+/-0.39)$ & $1.7(1.8+/-0.33)$ & 0.68 & $>0.5$ \\
\hline Pain score on day 3 & $2.03(1.903+/-0.34)$ & $3.4(2.4+/-0.45)$ & 2.2 & 0.01 \\
\hline
\end{tabular}

\subsection{Post-operative complications}

The post-operative complications were studied at the end of one year follow up. The complications were divided into major and minor. Patients requiring admission into the hospital and operative interventions were considered as major complications and the patients who were treated on outpatient basis were considered as minor complications.

\subsection{Major complications}

There was no statistical significant difference in complications between two groups $(p>0.05)$. At the end of one year follow up each group had one revision knee surgery. One patient in subvastus had open reduction and internal fixation with locking compression plate for peri prosthetic fracture of the tibia. Four patients in medial parapatellar group had reoperation for insertion of patella button. Both medial parapatellar and subvastus had one patient who underwent manipulation under anaesthesia of knee for stiffness

\subsection{Minor complications}

The patients who were managed as an outpatient without admission into hospital for all the complications were considered as minor complications. There was no statistical significant difference between two groups ( $p>0.05)$. In medial parapatellar group one patient had stitch abscess, two had cellulitis around knee and one patient had stiffness of knee which required physiotherapy. In subvastus group three patients had superficial wound infection and three developed blisters around operated knee joint.

Table 4: Minor complications

\begin{tabular}{|c|c|c|}
\hline Minor complication & Subvastus & Medial parapatellar \\
\hline Stitch abscess & 0 & 1 \\
\hline Cellulitis around knee & 0 & 2 \\
\hline Superficial wound infection & 3 & 0 \\
\hline Blisters around knee & 3 & 0 \\
\hline Physiotherapy for stiffness of knee & 0 & 1 \\
\hline
\end{tabular}

Table 5: Major Complications

\begin{tabular}{|c|c|c|}
\hline Major complications & Subvastus & Medial para patellar \\
\hline MUA of knee for stiffness & 1 & 1 \\
\hline ORIF of Tibia for peri prosthetic fracture & 1 & 0 \\
\hline Insertion of patella button & 0 & 4 \\
\hline Revision knee surgery & 1 & 1 \\
\hline
\end{tabular}

\section{Discussion}

In this study, it was found that the subvastus approach had better component alignment in sagittal plane of tibia, coronal plane of femur and tibia and the tibio femoral anatomical axis. No significant difference was found in joint line measurement between the two groups. These results are contrary to results found by Chen, et al. ${ }^{[13]}$, Pan, et al. ${ }^{[14]}$ and Lai, et al. ${ }^{[15]}$ who found component alignment was inferior in subvastus approach. But Weinhardt, et al. ${ }^{[16]}$, Hart, et al. ${ }^{[17]}$ Young, et al. ${ }^{[18]}$ and Dutka, et al. ${ }^{[19]}$ found no difference in the component alignment between subvastus and medial parpatellar approaches.

The acceptable range for tibia component alignment in coronal plane is 88 to 92 degrees to get better results and longer survival of the implant ${ }^{[20,21,22]}$. Mal alignment of the tibial component alters the distribution of tibial loading, which may result in increased shear forces along the tibio femoral interface, leading to increased wear. Tibial mal 
alignment of more than 3 degrees of varus will increase the risk of medial bone collapse (Berend et al. ${ }^{[23]}$. The coronal alignment of femur and tibia in this study were within normal acceptable range in both the groups. It was 90.57 degrees in subvastus and 88.48 in medial parapatellar group.

The component alignment of femur in coronal plane was within acceptable range in both the approaches in our study. The acceptable range is 4 to 11 degrees of valgus with optimal of 7 degrees ${ }^{[24,25]}$. The ideal femoral component positioning in sagittal plane is 83 degrees of flexion to 90 degrees of neutral alignment to improve the survival rate and functional outcome following total knee arthroplasty. Whereas, the accepted range of tibial component placement in sagittal plane is posterior tibial slope between 0 to 7 degrees, as placement of the tibial component beyond this range may lead to instability. If there was excessive posterior tibial slope it could lead to flexion gap tightness and reduced postoperative flexion if there was relative anterior tibial slope ${ }^{[11]}$. In this study the sagittal alignment of tibia and femur was better in subvastus approach. There were no significant difference between two groups in terms of blood loss and blood transfusions $(\mathrm{p}>0.05)$. This was similar to findings of meta-analysis by Teng, et al. ${ }^{[27]}$ and in randomised controlled studies by Weinhardt, et al. ${ }^{[16]}$, Bridgman, et al. ${ }^{[28]}$, and Bourke, et al. ${ }^{[29]}$. Whereas Roysam and Okaley ${ }^{[9]}$ and Chen, et al. ${ }^{[13]}$ in their study found that there was less blood loss and shorter tourniquet time in the subvastus group. In this study blood loss was measured as difference in preoperative haemoglobin and post-operative haemoglobin. It is a better method of measure of blood loss than quantifying methods of blood loss estimation, as our method of blood loss measurement was not subjected to observer error. The other methods of estimating blood loss like counting number of swabs used, drain collection does not include hidden blood loss [30].

Duration of surgery was significantly higher in subvastus group when compared to medial parapatellar approach ( $p$ value < 0.01). The other studies with similar results were by Bridgman, et al. ${ }^{[28]}$, Bourke, et al. ${ }^{[29]}$ and Lai, et al. ${ }^{[15]}$. However Teng, et al. [27] in their meta-analysis and Weinhardt, et al. ${ }^{[16]}$ in their randomised controlled study found no difference between two groups in terms of duration of surgery. This could be explained as it takes more time to expose the joint because of technical difficulties.

Pain score on day 3 was significantly higher in medial parapatellar group when compared to subvastus group, whereas on day 1 there was no significant difference between two groups. Roysam and Oakley [9] Bridgman, et al. [28] Dutka, et al. ${ }^{[19]}$ and Tomek, et al. ${ }^{[31]}$ found similar results. But, no difference in pain was found between two groups according to Weinhardt, et al. ${ }^{[16]}$, Wouter, et al. ${ }^{[32]}$ and Teng, et al. ${ }^{[27]}$ in their studies. We used visual analogue score for measurement of severity of pain in post-operative period. The drawback of this method was that the patient with higher intake of analgesia could have less pain score and may lead to bias in the results.

Patients who underwent subvastus approach had shorter stay in the hospital for 6.67 days (range 3-11 days) compared to 8.83 days (range 4-18 days) in medial parapatellar group. However it was not statistically significant $(\mathrm{p}>0.05)$. This was similar to results obtained by Bridgman, et al. (2008), Bourke, et al. (2012) ${ }^{[8]}$ and Teng, et al. (2012). But contrary to results found by Chen, et al. (2006). This shorter stay could be due to reduced pain in post-operative period and preservation of quadriceps mechanism in subvastus group and patients could have mobilised earlier in post-operative period. There was no statistical significant difference in postoperative complications between two groups over one year follow up. This was similar to studies done by Teng, et al. (2012), Dutka, et al. (2011) [13], Roysam and Okaley, (2001), Chen, et al. (2006) and Weinhardt, et al. (2004) ${ }^{[33]}$. But higher rate of complications in medial parapatellar group were found in Matsueda and Gustilo (2000) ${ }^{[26]}$ study.

\section{Conclusion}

Subvastus can also be considered as an alternative surgical approach along with standard medial parapatellar approach for primary total knee arthroplasty. It offers better results in terms of component alignment, less pain in early postoperative period without significant differences in the complications of surgery compared to standard approach provided the surgeon operating the subvastus approach has adequate experience.

\section{References}

1. Alastair Younger SE, Clive Duncan P, Bassam Masri A. Surgical Exposures in Revision Total Knee Arthroplasty Journal of American academic orthopaedic surgeons. 1998; 6:55-64.

2. Allen AM, Ward WG, Pope TL. Imaging of the total knee arthroplasty. Radiology Clinics of North American. 1995; 33(2):289-303.

3. Abbasi S, Khan FA, Adil S, Enam SA. Comparison of visual estimation of blood loss with serial hemoglobin and hematocrit estimation in supratentorial craniotomy. Priory Medical Journal, 2011, 1-10.

http://www.priory.com/anaesthesia/Blood-Loss.htm (accessed on 3/10/2013)

4. Berend ME, Ritter MA, Meding JB, Faris PM, Keating EM, Redelman R. Tibial component failure mechanisms in total knee arthroplasty. Clinical Orthopaedics, 2004; 428:26-34.

5. Bijur PE, Silver W, Gallagher EJ. Reliability of the visual analog scale for measurement of acute pain. Academic of Emergency Medicine. 2001; 8(12):1153-7.

6. Bourke MG, Buttrum PJ, Fitzpatrick PL, Dalton PA, Jull GA, Russell TG. Systematic review of medial parapatellar and subvastus approaches in total knee arthroplasty Journal of Arthroplasty. 2009; 25(5):728-34.

7. Bourke MG, Jull GA, Buttrum PJ, Fitzpatrick PL, Dalton PA, Russell TG. Comparing outcomes of medial parapatellar and subvastus approaches in total knee arthroplasty: a randomized controlled trial. Journal of Arthroplasty. 2012; 27(3):347-353.

8. Bridgman AS, Walley G, MacKenzie G, Clement D, Griffiths D, Maffulli N. Sub-vastus approach is more effective than a medial parapatellar approach in primary total knee arthroplasty: A randomized controlled trial'. Knee. 2008; 3:216-222.

9. Chen AF, Alan RK, Redziniak DE, Tria AJ. Quadriceps sparing total knee replacement. The initial experience with results at two to four years. Journal of Bone Joint Surgery Br. 2006; 88:1448-1453.

10. Cila E, Güzel V, Ozalay M, Tan J, Simşek SA, Kanatli U, et al. Subvastus versus medial parapatellar approach in total knee arthroplasty. Archieves of Orthopaedic Trauma Surgery. 2002; 122(2):65-8.

11. Dennis DA, Channer M, Susman MH, Stringer EA. Intramedullary versus extramedullary tibial alignment systems in total knee arthroplasty. Journal of 
Arthroplasty. 1993; 8(1):43-47.

12. Dutka J, Skowronek M, Sosin P, Skowronek P. Subvastus and medial parapatellar approaches in TKA: comparison of functional results. Orthopedics. 2011; 34(6): 148.

13. Ewald FC. The Knee Society total knee arthroplasty roentgeno graphic evaluation and scoring system' Clinical orthopaedic related research. 1989; 248:9-12.

14. Figgie HE, Goldberg VM, Figgie MP, Inglis AE, Kelly M, Sobel M. The effect of alignment of the implant on fractures of the patella after condylar total knee arthroplasty'. Journal of bone and Bone Joint Surgery. 1989; 71:1031-1039.

15. Freeman MA, Swanson SA, Todd RC. Total replacement of the knee using the Freeman-Swanson knee prosthesis'. Clinical Orthopaedic Related Research. 1973; 94:153170.

16. Gromovo K, Korchi M, Thomsen M, Husted H, Troelsen A. What is the optimal alignment of the tibial and femoral component in knee arthroplasty? An overview of the literature'. Acta orthopaedica journal. 2014; 85(5):480-487.

17. Hart R, Janecek M, Cizmar I, Stipcak V, Kucera B, Filan P. Minimal-invasive und navigierte Implantation von Knietotalendoprothesen. Radiologische analyse und frühe klinische Ergebnisse'. Orthopade. 2006; 35:552-557.

18. Hernandez VD, Gonzalez UJ, Fernandez CC, Sandoval MA, Gonzalez AR. Patellar complications after total knee arthroplasty'. International Orthopaedic journal. 1996; 20(2):103-106.

19. Hofmann AA, Plaster RL, Murdock LE. Subvastus (Southern) approach for primary total knee arthroplasty'. Clinical orthopaedics \& related research. 1991; 269:70-7.

20. Kane RL, Saleh KJ, Wilt TJ. Total knee replacement. Evidence Report Technology Assessment. 2003; 86:1-8.

21. Khan RJK, Keogh A, Fick D, Wood D. Surgical approaches in total knee arthroplasty'. (Protocol) Cochrane Database System Rev. 2005, 1,2.

22. Khanna A, Gougoulias N, Longo U, Maffulli N. Minimally Invasive Total Knee Arthroplasty: A Systematic Review'. Orthopedic Clinics of North America. 2009; 40(4):479-489.

23. Lai Z, Shi S, Fei J, Wei W. Total knee arthroplasty performed with either a mini-subvastus or a standard approach: a prospective randomized controlled study with a minimum follow-up of 2 years'. Archieves of Orthopaedic and Trauma Surgery. 2014; 134(8):1155-62.

24. Langenbeck V. Zur resection des kniegelenke. Verh' Dtsch En Geseuch F Chir. 1879; 7:23.

25. Matsueda M, Gustilo RB. Subvastus and medial parapatellar approaches in total knee arthroplasty' Clinical Orthopaedic Related Research. 2000; 371:161168.

26. National joint registry for England and Wales. $11^{\text {th }}$ Annual report 2014'. http://www.njrcentre.org.uk. (Accessed on 12/10/2014)

27. Pang J, Shen S, Pan R, Jones IR, Rozen WM, Taylor GI. The arterial supply of the patellar tendon: anatomical study with clinical implications for knee surgery'. Clinical Anatomy. 2009; 22(3):371-376.

28. Roysam GS, Oakley MJ. Subvastus approach for total knee arthroplasty: A prospective, randomized, and observer-blinded trial. Journal of Arthroplasty. 2001; 16(4):454-457.
29. Robertson O, Knutson K, Lewold S, Lidgren L. The Swedish Knee Arthroplasty Register 1975-1997: an update with special emphasis on 41,223 knees operated on in 1988-1997'. Acta Orthopaedic Scandinavia. 2001; 72:503-13.

30. Sarmah SS, Patel S, Hossain FS, Haddad FS. The radiological assessment of total and unicompartmental knee replacements. Jouranl of Bone Joint Surgery. 2012; 94(B):1321-29.

31. Selvarajah E, Hooper G. Restoration of the joint line in total knee arthroplasty. Journal of Arthroplasty. 2009; 24:1099-1102.

32. Weinhardt C, Barisic M, Bergmann EG, Heller KD. Early results of subvastus versus medial parapatellar approach in primary total knee arthroplasty'. Archives of Orthopaedics Trauma and Surgery. 2004; 124(6):401-3.

33. Wouter LW van Hemert, Rachel Senden, Bernd Grimm, Matthijs JA van der Linde, Arno Lataster, Ide C. Heyligers. Early functional outcome after subvastus or parapatellar approach in knee arthroplasty is comparable: a performance-based trial with anatomical findings. Knee Surgery, Sports Traumatology, Arthroscopy. 2011; 20(9):885-1886.

34. Young B, Jung Y, Lee S, Yong E, Lee H, Joong J, et al. Comparison of the modified subvastus and medial parapatellar approaches in total knee arthroplasty' International Orthopaedics. 2009; 33(2):419-423. 